\title{
Direct Numerical Simulation of Liquid Films with Large Interfacial Deformation ${ }^{\dagger}$
}

\author{
By J.-C. Nave, X. D. Liu, and S. Banerjee
}

Liquid films are important in many industrial applications, but also from a fundamental point of view, they are important two-phase flow systems. In this paper, we develop a sharp interface/level set method for the Direct Numerical Simulation (DNS) of liquid films with large interfacial deformations, and large density ratio between the liquid and the gas phase. We use the ghost fluid method to capture the interface motion without smoothing properties across it, and adopt a maximization scheme for the implicit treatment of the viscous term in the Navier-Stokes equations. Because liquid films have very low average depth compared to the distance between waves, several innovations are required to handle solving the equations on grid structures of high aspect ratio. Two-dimensional (2D) calculations for wavy films falling down a vertical wall are presented, and good agreement is found when numerical solutions are directly compared with the experiments of Nosoko et al. [1]. Some results are also presented for falling liquid films transitioning naturally from $2 \mathrm{D}$ to $3 \mathrm{D}$ surface wave structures demonstrating the potential of the method for 3D fully coupled two-phase liquid films simulations.

\section{Introduction}

The overall focus of the present work is to develop a method for directly simulating two-phase flows, that is capable of handling large interfacial

\footnotetext{
Address for correspondence: J.-C. Nave, Department of Mathematics, Massachusetts Institute of Technology, 77 Massachusetts Avenue 2-363A, Cambridge, MA 02139, USA; e-mail: jcnave@mit.edu ${ }^{\dagger}$ Dedicated to the memory of $\mathrm{Xu}$ Dong, our friend and colleague.
} 


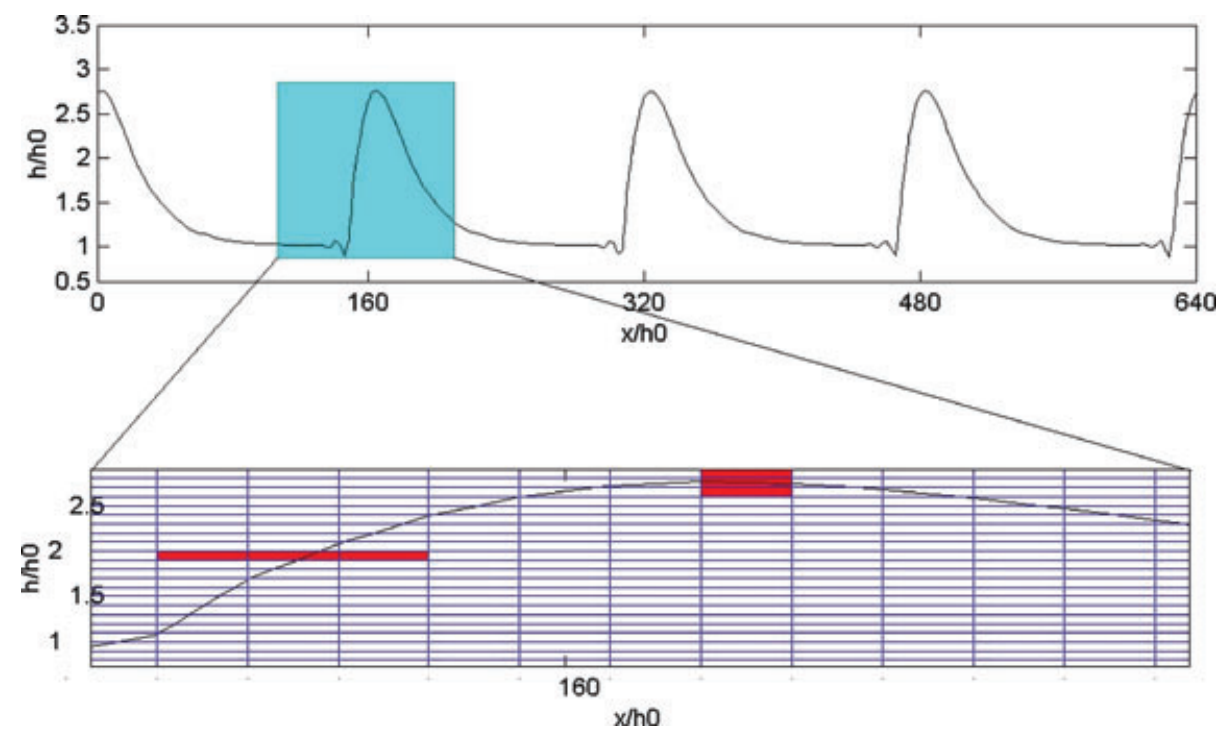

Figure 1. Effect of smeared interface formulation for an elongated grid. This figure is colour online.

deformations, and allows changes in interfacial topology, for example, wave breaking and/or droplet detachment. Also, the approach must be able to treat large surface tensions and large density ratios while maintaining a "sharp" interface. Liquid films have in general a large aspect ratio, thus requiring long computational domains. Consequently, we investigate whether simulating a small number of wave lengths (allowing for higher resolution) using periodic boundary conditions accurately predicts film statistics such as those presented in [1]. To these ends, we chose to use the finite difference method on a Marker And Cell (MAC) grid which allowed us to benefit substantially from the work of previous investigators [2-5].

Specifically, the simulation of falling liquid films is a particularly demanding problem; typically, falling liquid films have large aspect ratio. The ratio of the wavelength to the average film thickness may be 100 or more. Aside from the resolution requirements associated with simulations on high aspect ratio domains, the waves may present relatively steep fronts (see Figure 1). Consequently, any numerical method must be able to accurately capture the interface in both the wall-normal direction as well as in the flow direction. These requirements imply that traditional simulation techniques must use square (or slightly elongated) meshes. This results in large problems that are often too demanding for current computational capabilities. For a typical film flow simulation with 100 grid points in the wall-normal direction, and a domain size of four wave lengths, the simulation would require $100 \times 20000 \times 20000$ 
(roughly cubical) grid points, which is out of reach of common computer systems. As a result, most simulation must be conducted on grids with large aspect ratios, where $\Delta y \approx \Delta z \approx 100 \Delta x$ for a 100:1 aspect ratio film.

With a finite-difference approach, several methods used for the simulation of two-phase flows assume a smeared interface representation. The smeared interface approach [6], refers to approximating the discontinuous quantities (fluid densities, viscosities) with their smooth analogs typically over a distance $\Delta \epsilon=3 \max (\Delta x, \Delta y, \Delta z)$. While this approach is valid and accurate in most physical situations for which grids with aspect ratios near unity are adequate, using an elongated grid of aspect ratio $\gamma=\max (\Delta x, \Delta y, \Delta z) / \min (\Delta x, \Delta y, \Delta z)$ would translate into smearing the interface over up to $\Delta \epsilon \cdot \gamma$ grid points in at least one direction. The interface smearing leads to problems when the film surface is nearly vertical as is the case for the wave front in Figure 1; on the front part of the wave, when the interface is nearly perpendicular to the wall, the smearing is performed over a distance larger by $\gamma$ compared to the other direction. This effect introduces errors, and becomes problematic for the study of processes where interface-local quantities are of interest such as heat transfer and mass transfer.

Another approach [7] is to treat the interface as a contact surface, maintaining a "sharp" interface. This approach assumes piece-wise constant properties (viscosity, density) on each domain and matches the two sides using appropriate boundary conditions and locally 1D interpolation. This method eliminates the issues associated with the smeared interface representation. As a result, the need for resolution dictated by the interface region is reduced at the expense of a more complicated formulation. Additionally, solving for pressure through the variable coefficient Poisson equation, an elliptic problem with jumps discontinuous interface conditions, becomes more involved as surface tension forces must be included as a pressure jumps rather than a smooth transition across the interface.

The problem associated with discretizing the equation for the pressure with interfacial jump conditions was addressed in [8]. The approach for solving the Navier-Stokes equations in rectangular coordinates with two phases and including surface tension, was developed by Kang et al. [2] and was named the Ghost Fluid Method (GFM) in [7]. The method described in [2] is fully explicit, and did not consider accuracy for large aspect ratio grids, semi-implicit treatment of the viscous term, or turbulence. In the present work we address the first two issues, and motivate the appropriateness of the method for the future study of two-phase turbulence.

This paper is organized in the following way: in Section 2 we review the background on liquid films and previous numerical work, Section 3 presents the governing equations, and describes in detail the present scheme, in Section 4 we validate our approach by directly comparing our numerical results 
with experiments, finally in Section 5 we summarize our main contribution, and provide an outlook toward possible future research.

\section{Objectives and background}

As mentioned earlier, the objective of this study is to develop a numerical method suitable for the study of a wide range of two-phase flows and validate it for liquid films that are either gravity, or gas driven. The numerical scheme also should be efficient, accurate, and able to handle arbitrarily large surface tension forces, density ratios, and deformations of the interface. We will now briefly discuss previous work with somewhat similar goals that have been used for the simulation of free surface, or two-phase systems, in the context of the objectives stated earlier.

The objectives outlined earlier are most closely met by [2]. Improvements and validation to this approach are, however, necessary:

(1) Implicit treatment of the viscous term allowing us to remove the strong stability restriction on step size associated with the fully explicit schemes presented in [2]. This is especially important because wall-normal mesh spacing is typically one order of magnitude smaller than the stream-wise mesh spacing due to the nature of the problem.

(2) Validation of simulating liquid films systems using periodic boundary conditions. The periodic treatment is necessary for the simulation of turbulence, and to replace the spatially elongated systems that are required for inflow/outflow boundary conditions to obtain stationary flow statistics.

One of the first numerical schemes developed for the simulation of free surface flows is due to Harlow and Welch [9]. In their work, they used a MAC grid approach and a fractional step method later formalized by Chorin [10]. With the increase in computational power, and the efficiency of the Fast Fourier Transform (FFT), a number of schemes were developed to be able to simulate larger problems, including turbulence. Many such schemes, referred to as "pseudo spectral methods" are referenced in [11]. For example, the problem of flat free surface turbulence has been extensively studied in [12], and [13].

For general two-phase flow computations, only a few methods can handle surface tension forces and large density ratios. In the Volume of Fluid (VoF) method, Hirt and Nichols [14] use piecewise functions (often linear) to reconstruct the interface locally and determine the fraction of one phase, that is within a cell. This method has recently been applied to the simulation of 2D wavy falling liquid films with a free surface [15]. However, it only calculates the volume fraction occupied by each phase in an interfacial cell so the shape of the interface has to be reconstructed. Another method uses a level 
set function $\phi$, to implicitly determine the position of the interface as its zero contour. As a result, fluid properties such as viscosity and density are defined as a function of $\phi$

$$
\begin{aligned}
& \mu(\phi)=\mu^{-}+\left(\mu^{+}-\mu^{-}\right) H(\phi), \\
& \rho(\phi)=\rho^{-}+\left(\rho^{+}-\rho^{-}\right) H(\phi),
\end{aligned}
$$

where $H(\phi)$ is a Heaviside function, and the "+" and "-" superscripts define the two different phases, liquid and gas, respectively.

Surface tension forces are included using the Continuum Surface Force (CSF) model [16]. In this formulation, surface tension enters as a source term in the momentum equation and is located at the interface through the use of a delta function in $\phi$,

$$
\delta(\phi) \equiv \frac{d H}{d \phi} .
$$

Because in the Eulerian framework, equations are discretized on a grid, the delta function (2), and the Heaviside functions in (1) must be regularized to avoid $\mathcal{O}(0)$ discrete effects. Consequently, most studies suggest smearing (1), and (2) over a distance $\epsilon$. As a result, and taking into account that the delta function should integrate to 1 , we define

$$
\begin{gathered}
\delta_{\epsilon}(\phi)= \begin{cases}\frac{1}{2 \epsilon}\left[1+\cos \left(\frac{\pi \phi}{\epsilon}\right)\right], & |\phi| \leq \epsilon, \\
0, & |\phi|>\epsilon,\end{cases} \\
H_{\epsilon}(\phi)= \begin{cases}0, & \phi<-\epsilon, \\
\frac{1}{2}\left[1+\frac{\phi}{\epsilon}+\frac{1}{\pi} \sin \left(\frac{\pi \phi}{\epsilon}\right)\right], & |\phi| \leq \epsilon, \\
1, & \phi>\epsilon .\end{cases}
\end{gathered}
$$

We can verify directly that,

$$
\int_{-\infty}^{+\infty} \delta_{\epsilon}(\phi) d \phi=1
$$

The method of using smeared quantities is appropriate in most situations where the aspect ratio of the physical problem is nearly one. However, as noted earlier, this method is not adequate for the simulation of large aspect ratio problems such as falling liquid films. 


\section{The level set/ghost fluid method approach}

We now turn our attention to the sharp interface method for the simulation of falling liquid films, the Ghost Fluid Method (GFM) of Kang et al. [2] and Liu et al. [8].

Problems associated with the simulation of falling liquid films are demanding and constitute a good test of any proposed method. Specifically, the following issues will be addressed in this section for the development of the numerical scheme.

First, falling liquid films have typically elongated aspect ratios; the wavelengths may be one hundred times the thickness of the film. This forces the use of elongated meshes in the direction of the flow to make for a practically tractable problem size. Typically, this translates into having an aspect ratio of 10:1 for the mesh, which in turn gives roughly 10 times more grid points in the streamwise direction compared to the wall normal direction. As an aside, such meshes elongated in the streamwise direction are also desirable for wall turbulence simulations as streamwise structures, such as quasi-streamwise vortices, are much longer than their extent in wall normal or spanwise directions. Therefore, such elongated meshes are also necessary for the study of turbulent liquid films - a subject of great practical interest. These two adjustments can account for a wave length to film thickness ratio of $\sim 100: 1$ which is observed in experimental studies [1]. Also, falling liquid films may exhibit large curvatures and nearly vertical wave fronts we use the GFM for its capacity to treat sharp interfaces, and to accurately model surface tension forces at the sub-grid level. The GFM allows the interface to be defined to first-order accuracy independently of its local orientation with respect to the grid.

Second, for a film to reach statistically steady state conditions requires long simulation domains if inflow/outflow boundary conditions are used [17]. As a result, accurate simulations of large wavelength problems using inflow/outflow boundary condition are prohibitively expensive. To circumvent this problem we propose to use periodic boundary conditions with an initial, spatial wavelength disturbance. In effect, the film develops as a transient problem in the periodic domain, rather than over a long spatial domain with inflow/outflow boundaries. This is of course a very common procedure for turbulence simulations. We will verify the validity of this assumption in Section 4 in the context of falling liquid films simulations.

Third, many problems of interest exhibit a wide range of scales in liquid films flows. For instance, the amplitude of capillary waves can be an order of magnitude smaller than that of a solitary wave. This observation requires using small grid spacing to correctly capture the physics present at the capillary wave scale. As a result, the explicit treatment of viscosity in the GFM [2] imposes stability conditions on time step sizes that are too restrictive even for laminar problems of this nature. Note that this restriction occurs because 
the scales present in the problem are small in the wall normal direction (capillary waves). In contrast to single phase homogeneous turbulence, for which in general an explicit treatment of the viscous term suffices, the need in two-phase flows of resolving small interfacial features requires an implicit scheme for the viscous term. We propose an implicit treatment of viscosity through a maximization approach similar to that of Badalassi et al. [18]. This approach relaxes the restriction on the time step and allows us to perform simulations with higher spatial resolution at the same computational cost.

\subsection{Problem setup and governing equations}

The physical setup that must be addressed by the numerical scheme is shown in Figure 2. We use periodic boundary conditions in the $y$-direction, no slip walls in the $x$-direction, and slip walls in the $z$-direction in all three-dimensional cases. The basic equations for an incompressible viscous fluid are the Navier-Stokes equations. In rectangular coordinates,

$$
\begin{gathered}
\rho_{t}+\nabla \cdot(\rho V)=0, \\
\rho\left(V_{t}+(V \cdot \nabla) V\right)=-\nabla p+(\nabla \cdot \tau)^{T}+F,
\end{gathered}
$$

where ". $T$ " represents the transpose, $F$ the body forces, $\tau=\frac{1}{2} \mu\left[\nabla V+(\nabla V)^{T}\right]$ is the viscous stress tensor for incompressible viscous Newtonian fluid, and $V=\langle u, v, w\rangle$. The density $\rho$ and the viscosity $\mu$ are both functions of $\phi$, the signed distance from the interface, and are defined in the following "sharp" manner,

$$
\begin{array}{r}
\rho(\phi)= \begin{cases}\rho_{\text {liquid }}, & \phi>0, \\
\rho_{\text {gas }}, & \phi \leq 0,\end{cases} \\
\mu(\phi)= \begin{cases}\mu_{\text {liquid }}, & \phi>0, \\
\mu_{\text {gas }}, & \phi \leq 0 .\end{cases}
\end{array}
$$

The level set function $\phi$ is obtained by solving the following equation

$$
\phi_{t}+V \cdot \nabla \phi=0
$$

From $\phi$, we can compute the normal and curvature

$$
\begin{gathered}
N=\frac{\nabla \phi}{|\nabla \phi|}, \\
\kappa=-\nabla \cdot N .
\end{gathered}
$$

Following the GFM formulation, we include surface tension forces as an internal jump condition for the pressure. 


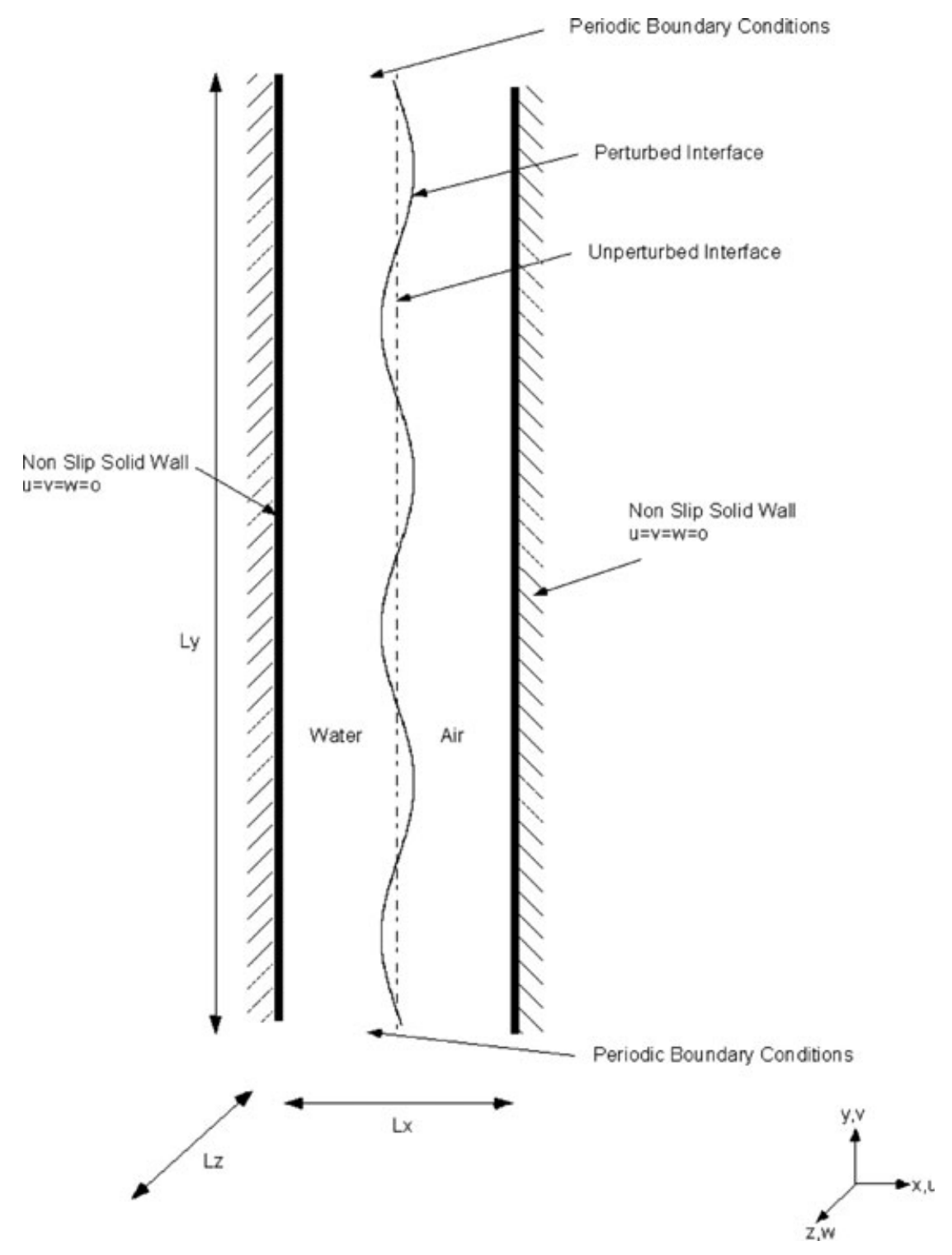

Figure 2. Physical setup.

\subsection{Numerical method}

A staggered grid formulation is used to discretize the basic equations. Velocity components are defined on cell faces, while the pressure, and level set function are defined at the center of the control volume. The solver for the Navier-Stokes equation is an evolution of the scheme in [2]. We used a semi-implicit time marching method with a second-order Adams-Bashforth method for the 
explicit terms and a maximization scheme for the implicit terms. A fractional step method, projection method [10] is used to solve the Navier-Stokes equation. The variable density and viscosity are treated "sharply" and jump conditions are computed using sub-grid linear interpolation based on the level set function $\phi$. The discretized equations for conservation of momentum are

$$
\begin{aligned}
\frac{V^{n+1}-V^{n}}{\Delta t}= & -\frac{\nabla p^{n+1}}{\rho}+\frac{3}{2}\left[-(V \cdot \nabla) V+\frac{F}{\rho}-\frac{v_{\max }}{2} M\right]^{n} \\
& -\frac{1}{2}\left[-(V \cdot \nabla) V+\frac{F}{\rho}-\frac{v_{\max }}{2} M\right]^{n-1}+\left[\frac{(\nabla \cdot \tau)^{T}}{\rho}\right]^{n} \\
& +\frac{v_{\max }}{2} M^{n+1}
\end{aligned}
$$

$$
\nabla \cdot V^{n+1}=0
$$

where, $M=\Delta V$ and $v_{\max }=\max \left(\frac{\mu_{\text {gas }}}{\rho_{\text {gas }}}, \frac{\mu_{\text {liquid }}}{\rho_{\text {liquid }}}\right)$.

Using the projection method, we define the following fractional steps,

$$
\begin{aligned}
\frac{V^{\star}-V^{n}}{\Delta t}= & \frac{3}{2}\left[-(V \cdot \nabla) V+\frac{F}{\rho}-\frac{\nu_{\max }}{2} M\right]^{n} \\
& -\frac{1}{2}\left[-(V \cdot \nabla) V+\frac{F}{\rho}-\frac{v_{\max }}{2} M\right]^{n-1} \\
& +\left[\frac{(\nabla \cdot \tau)^{T}}{\rho}\right]^{n}+\frac{v_{\max }}{2} M^{\star} \\
& \frac{V^{n+1}-V^{\star}}{\Delta t}+\frac{\nabla p^{n+1}}{\rho}=0 .
\end{aligned}
$$

Assuming that the velocity field at time level $n+1$ is divergence-free (14), we derive the variable density pressure Poisson equation

$$
\nabla \cdot\left(\frac{\nabla p^{n+1}}{\rho}\right)=\frac{\nabla \cdot V^{\star}}{\Delta t} .
$$

We assume no flow-through and no-slip conditions at solid wall boundaries, $V \cdot N=0$, and $V \times N=0$ respectively. The pressure boundary conditions at solid wall boundaries are Neumann, $\nabla p \cdot N=0$.

In the GFM, the treatment of property discontinuities (density, viscosity) at the interface requires jump conditions for the viscous term in the momentum equation (15), and in the Poisson equation (17). Following the notation in [2] 
we denote the jump across the interface by [·], and we compute the matrix of derivative jumps due to the discontinuity in viscosity,

$$
\begin{aligned}
\left(\begin{array}{ccc}
{\left[\mu u_{x}\right]} & {\left[\mu u_{y}\right]} & {\left[\mu u_{z}\right]} \\
{\left[\mu v_{x}\right]} & {\left[\mu v_{y}\right]} & {\left[\mu v_{z}\right]} \\
{\left[\mu w_{x}\right]} & {\left[\mu w_{y}\right]} & {\left[\mu w_{z}\right]}
\end{array}\right)= & {[\mu]\left(\begin{array}{c}
\nabla u \\
\nabla v \\
\nabla w
\end{array}\right)\left(\begin{array}{c}
0 \\
T_{1} \\
T_{2}
\end{array}\right)^{T}\left(\begin{array}{c}
0 \\
T_{1} \\
T_{2}
\end{array}\right) } \\
& +[\mu] N^{T} N\left(\begin{array}{c}
\nabla u \\
\nabla v \\
\nabla w
\end{array}\right) N^{T} N \\
& -[\mu]\left(\begin{array}{c}
0 \\
T_{1} \\
T_{2}
\end{array}\right)^{T}\left(\begin{array}{c}
0 \\
T_{1} \\
T_{2}
\end{array}\right)\left(\begin{array}{c}
\nabla u \\
\nabla v \\
\nabla w
\end{array}\right)^{T} N^{T} N,
\end{aligned}
$$

where $T_{1}$ and $T_{2}$ are the orthogonal and bi-orthogonal directions to the normal vector $N$. By knowing the values of the jumps across the interface, we can evaluate smoothly the individual derivatives on each side of the interface. More details on the derivation and discretization of the explicit part of the viscous term are available in [2].

The momentum equation can then be solved using an approximate factorization technique. We use standard central differences for the Laplacian terms in (15), WENO fifth order for the convection term, and the technique developed in [2] and [19] for the discretization of the divergence of the rate of strain tensor, $\tau$. For the solution of $V^{\star}$, we solve three tri-diagonal linear systems, one per dimension. Additionally, the implicit treatment of the viscous term by the maximization technique is intended to remove the $\mathcal{O}\left(\Delta x^{2}\right)$ stability restriction on the time step $\Delta t$.

Surface tension effects are incorporated into the equations as a jump in pressure across the interface. Thus, the jump condition for the pressure is

$$
[p]-2[\mu](\nabla u \cdot N, \nabla v \cdot N, \nabla w \cdot N) \cdot N=\sigma \kappa,
$$

where $\sigma$ is the surface tension, and is assumed to be constant in the present study.

The interface is evolved using the level set equation (10) which is discretized as

$$
\frac{\phi^{n+1}-\phi^{n}}{\Delta t}+V \cdot \nabla \phi^{n}=0
$$

where WENO fifth order is used to approximate $\nabla \phi$.

To preserve the level set function as a proper signed distance from the interface $(|\nabla \phi|=1)$, we use the reinitialization procedure with constraint from 
Sussman and Fatermi [20]

$$
\begin{aligned}
\phi_{\tau} & =\operatorname{sign}\left(\phi_{0}\right)(1-|\nabla \phi|)+\lambda f(\phi) \\
& \equiv L\left(\phi_{0}, \phi\right)+\lambda f(\phi),
\end{aligned}
$$

where $\phi_{0}$ is the level set function at pseudo-time $\tau=0$, and $\lambda$ is given by

$$
\lambda=\frac{\int_{\Omega} H^{\prime}\left(\phi_{0}\right) L\left(\phi_{0}, \phi\right)}{\int_{\Omega} H^{\prime}\left(\phi_{0}\right) f\left(\phi_{0}\right)},
$$

and where, $H(\phi)$ is the Heaviside function, $H^{\prime}(\phi)=d H(\phi) / d \phi$, and $f(\phi)=H^{\prime}(\phi)|\nabla \phi|$.

Equation (21) with $\lambda=0$ is the original reinitialization equation. We note that the steady state of (21) with $\lambda=0$ is $|\nabla \phi|=1$, the property we seek for the level set function. However, numerical errors cause the zero-contour of the level set function to be displaced during this procedure, leading to the loss (gain) of mass. As a result, Sussman and Fatermi [20] introduced the integrating factor $\lambda$, defined by (22) which represents a mass constraint for equation (21), thus guaranteeing that each cell domain $\Omega$ conserves the same mass throughout the procedure.

The integrations in (22) is performed using a second-order accurate Simpson's rule [21], over each individual grid cell $\Omega$ of the domain. Spatial derivatives in (21) are discretized using WENO fifth order, and the function is advanced in time using a third-order Runge-Kutta TVD scheme. Characteristics of (21) are normal outward to the contour $\phi=0$, thus the integration is performed for a few steps in pseudo time, guaranteeing accuracy of the solution on a strip around the interface. Additional details about the solution of the level set and reinitialization equations are available in [20].

The variable coefficient Poisson equation (17) is solved with the interface jump condition defined by (19). The discretization closely follows that described in [8]. To compute curvature around the interface, we use sub-grid linear interpolation

$$
\kappa_{\text {Int }}=\theta \kappa_{\text {Right }}+(1-\theta) \kappa_{\text {Left }},
$$

where $\theta$ is the cut cell defined by

$$
\theta=\frac{\left|\phi_{\text {Left }}\right|}{\left|\phi_{\text {Left }}\right|+\left|\phi_{\text {Right }}\right|} .
$$

This discretization produces a linear system $A p=b$ to be solved. Because the matrix $A$ is poorly conditioned, solving for pressure requires roughly $80 \%$ of the computational time. As a result, the solution method needs to be 


\section{Table 1}

Solution Scheme for 1 Time Step. (1 Euler Step, $E(V)$, is 1-4 Only)

1. Solve (15) for $V^{\star}$

2. Solve (17) for $p^{n+1}$

3. Solve (16) for $V^{n+1}$

4. Solve (20) for $\phi^{n+1}$ (Advection)

5. Solve (21) for $\phi^{n+1}$ (Reinitialization)

complemented by a preconditioner in order to limit the number of iterations required.

After investigating several iterative methods and preconditioners, we settled on the combination yielding the best robustness and efficiency for the simulation of liquid films. We use the BiCG-Stab iterative method from Van der Vorst [22] along with an Incomplete Upper-Lower (ILU) preconditioner. Note that the matrix $A$, produced from the discretization of the Poisson equation for the pressure is symmetric; however, we chose BiCG-Stab, an iterative method capable of solving non-symmetric systems, foreseeing evolving the method toward the solution of problems with irregular wall boundaries possibly resulting in nonsymmetric stencils.

Boundary conditions for most problems of interest in the present study have solid walls in $x$ and $z$ directions and are periodic in the $y$ direction. This results in a nonempty null space for $A$. We can therefore specify as an additional constraint that the sum of the residual be zero every time it is computed by subtracting a constant $c_{s}$

$$
\begin{aligned}
r_{\text {new }} & =r-c_{s}, \\
c_{s} & \equiv \frac{1}{n} \sum_{i=1}^{n} r_{i} .
\end{aligned}
$$

This guarantees convergence of the iterative solution for the Poisson equation. The entire solution scheme (Table 1), corresponding to an Euler step, with the exception of the reinitialization is then embedded in the third-order Runge-Kutta TVD scheme [23]

$$
V^{n+1}=\frac{1}{3} V^{n}+\frac{2}{3} E\left\{\frac{3}{4} V^{n}+\frac{1}{4} E\left[E\left(V^{n}\right)\right]\right\} .
$$




\subsection{Stability}

The original Ghost Fluid Method used an explicit discretization for the viscous term. We use a maximization scheme to relax the severe limitation imposed on the time step $\Delta t$ when the mesh size is reduced. Kang et al. [2] showed the limitation on the time step in the GFM to be

$$
\Delta t \leq\left(C_{c f l}+V_{c f l}+\sqrt{\left(C_{c f l}+V_{c f l}\right)^{2}+4 G_{c f l}^{2}+4 S_{c f l}^{2}}\right)^{-1},
$$

with

$$
\begin{aligned}
C_{c f l} & =\frac{\max (|u|)}{\Delta x}+\frac{\max (|v|)}{\Delta y}+\frac{\max (|w|)}{\Delta z}, \\
V_{c f l}^{E} & =\max \left(v^{-}, v^{+}\right)\left(\frac{2}{\Delta x^{2}}+\frac{2}{\Delta y^{2}}+\frac{2}{\Delta z^{2}}\right), \\
G_{c f l} & =\sqrt{\frac{|g|}{\Delta y}}, \\
S_{c f l} & =\sqrt{\frac{\sigma}{\min \left(\rho^{-}, \rho^{+}\right) \min (\Delta x, \Delta y, \Delta z)^{3}}} .
\end{aligned}
$$

For the maximization scheme, Badalassi et al. [18] showed

$$
V_{c f l}^{M}=\left[C\left(\frac{\max \left(\mu^{-}, \mu^{+}\right)}{\min \left(\mu^{-}, \mu^{+}\right)}-1\right)^{-1} \min (\Delta x, \Delta y, \Delta z)\right]^{-1},
$$

where $C$ is a constant.

To demonstrate stability of the new scheme, we used a 2D laminar falling liquid film problem at $R e=30$ where surface tension has been chosen to be low to remove the restriction imposed by $S_{c f l}$ in (28). We integrated the equations with different values of $\Delta x$ (mesh size), and chose for simplicity the same grid spacing in the $y$-direction. The solution was computed until steady state of the film or until numerical blowup due to violation of the stability constraint was observed. For each $\Delta x$, Figure 3 reports $\Delta t_{\max }$ for both the explicit scheme and the maximization scheme. The lines fitting the computed points are extrapolated to show the advantage of the maximization scheme for fine grid spacing. The extrapolation is based on the theoretical slopes for the maximization and explicit schemes with the constant being determined empirically from the data.

For the maximization approach used here, we observe proportionality to $\Delta x$ and not $\Delta x^{2}$, which is used for the explicit scheme data. We additionally checked that the solution for critical time steps in both cases reach the same statistically steady state wave profile and flow rates, that is, give essentially the same results. 


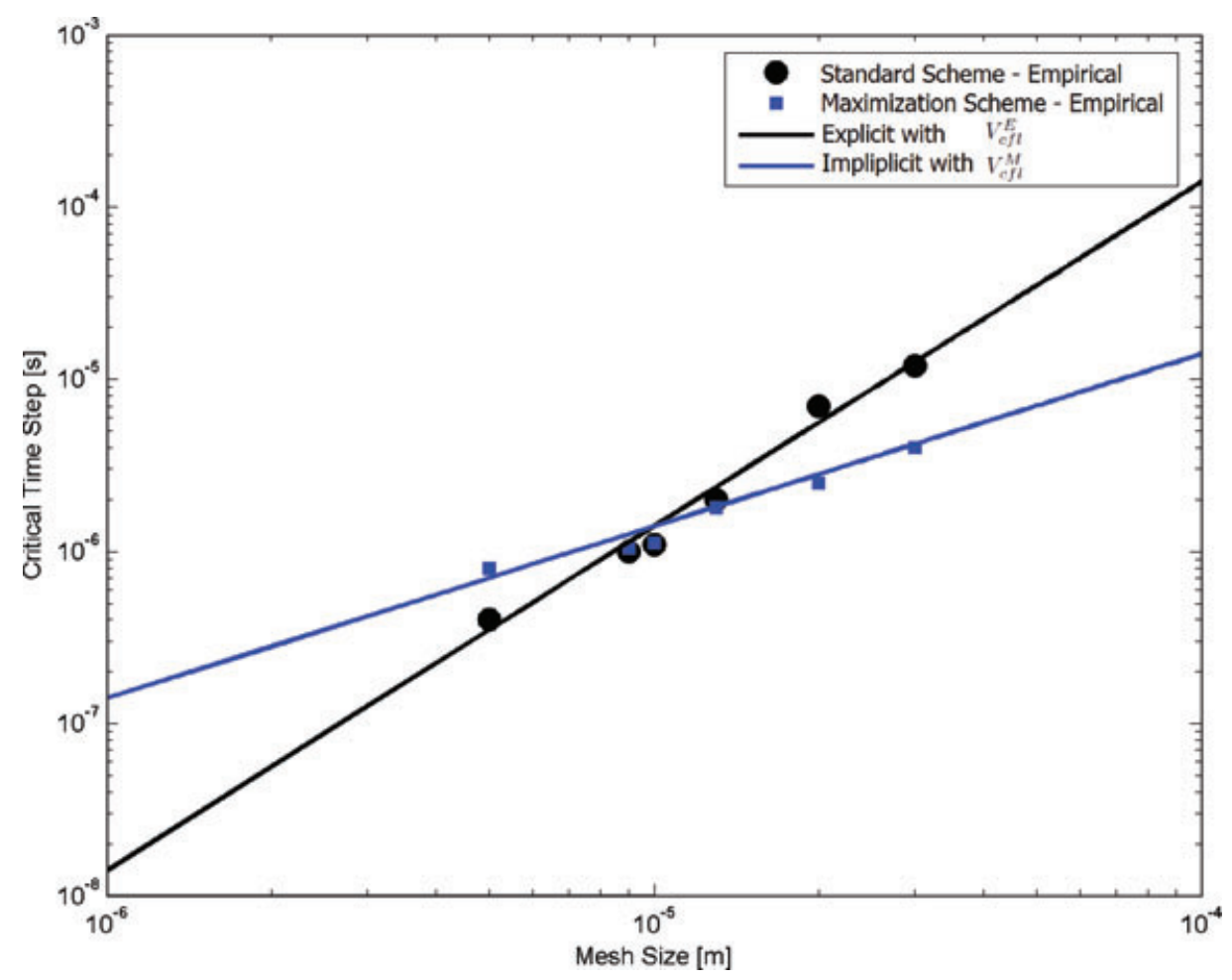

Figure 3. Critical stability comparison between the explicit GFM (black) and the maximization scheme (blue). Note that a mesh size of $5 \mathrm{e}-6$ corresponds to a $100 \times 100 \times 100$ grid for typical falling liquid film problems. This figure is colour online.

\section{Validation}

In this section, we focus on the simulation of vertically falling liquid films in the wavy-laminar regime in two-dimensions. First, definitions and the cases studied will be presented. Second, a quantitative study of the flow statistics will be compared with experiments to serve as validation of our approach.

\subsection{Numerical simulation and definitions}

As stated earlier, the aspect ratio for these films is very large. As a result, we performed our computation on a $2 \mathrm{D}$ grid of $50 \times 250$ mesh points for the range of Reynolds numbers from 30 to 100 . The $50 \times 250$ grid was chosen to guarantee convergence of the results. Similar computations were performed using inflow/outflow boundary conditions where inlet pressure is varied at a given frequency [17]. In the present study, we use periodic boundary conditions without temporal forcing. To set the scale of the problem, we define $\lambda_{s}$ as the initial conditions for the wavelength of the solitary wave and $N_{s}$ the number of 
Table 2

Computation Case Matrix

\begin{tabular}{lcccccc}
\hline & I & II & III & IV & V & VI \\
\hline $\operatorname{Re}$ & 103 & 103 & 69 & 50 & 40 & 30 \\
$N_{f} \cdot 10^{-12}$ & 0.41 & 0.052 & 0.2785 & 0.2018 & 0.1614 & 0.1614 \\
$N_{\lambda}$ & 994.9 & 994.9 & 826.8 & 742.6 & 689.4 & 689.4 \\
\hline
\end{tabular}

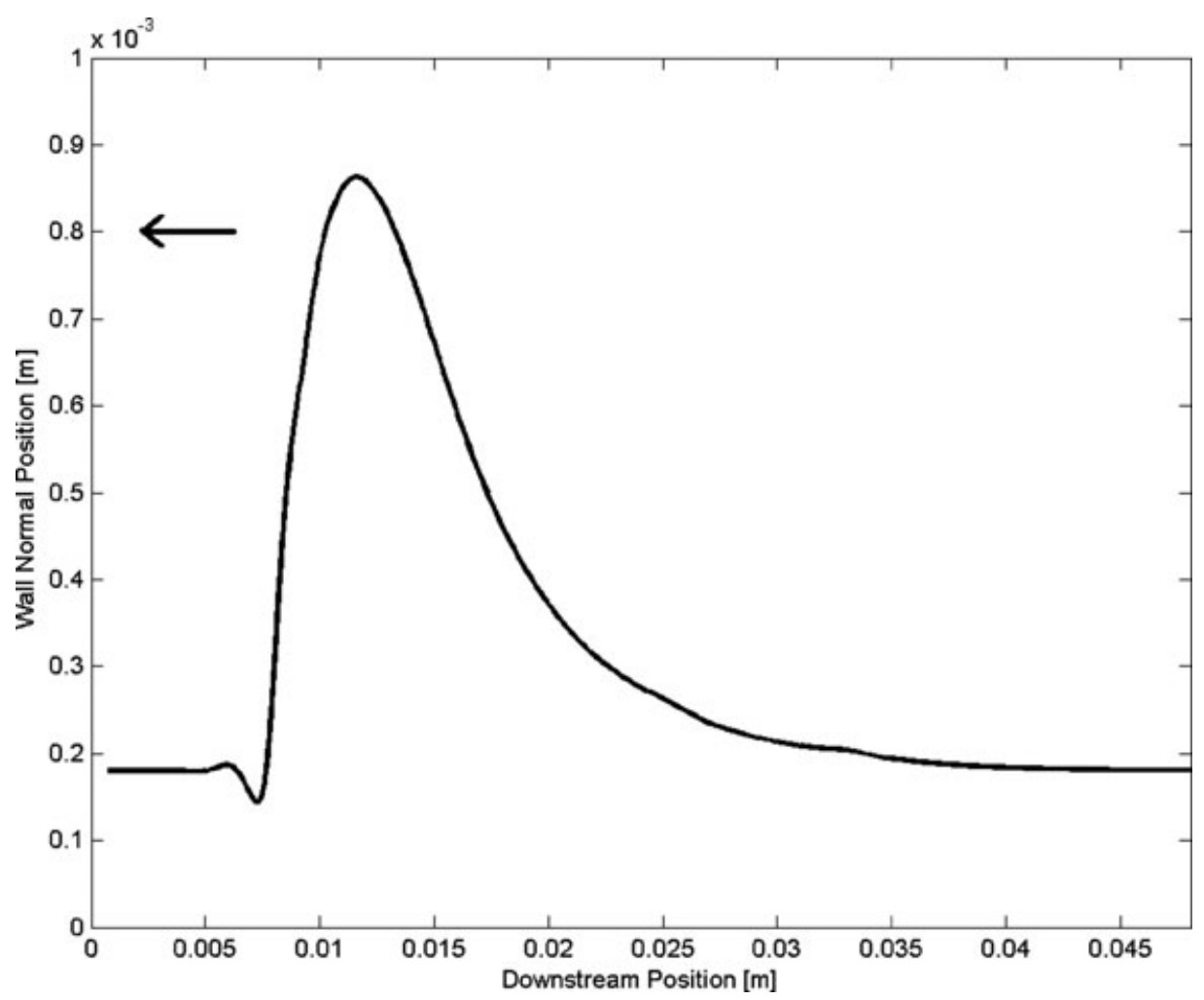

Figure 4. Typical wave profile corresponding to statistically steady state for case II.

waves per computational domain of length $L_{y}$. Here, $\lambda_{s}$ is chosen so that it corresponds to the steady-state solitary wave length observed in experiments. Also, the Reynolds number is defined based on the undisturbed film thickness $h_{0}$. We define the initial film thickness as

$$
h(y)=h_{0}+\epsilon \sin \left(\frac{2 \pi N_{s} y}{L_{y}}\right),
$$




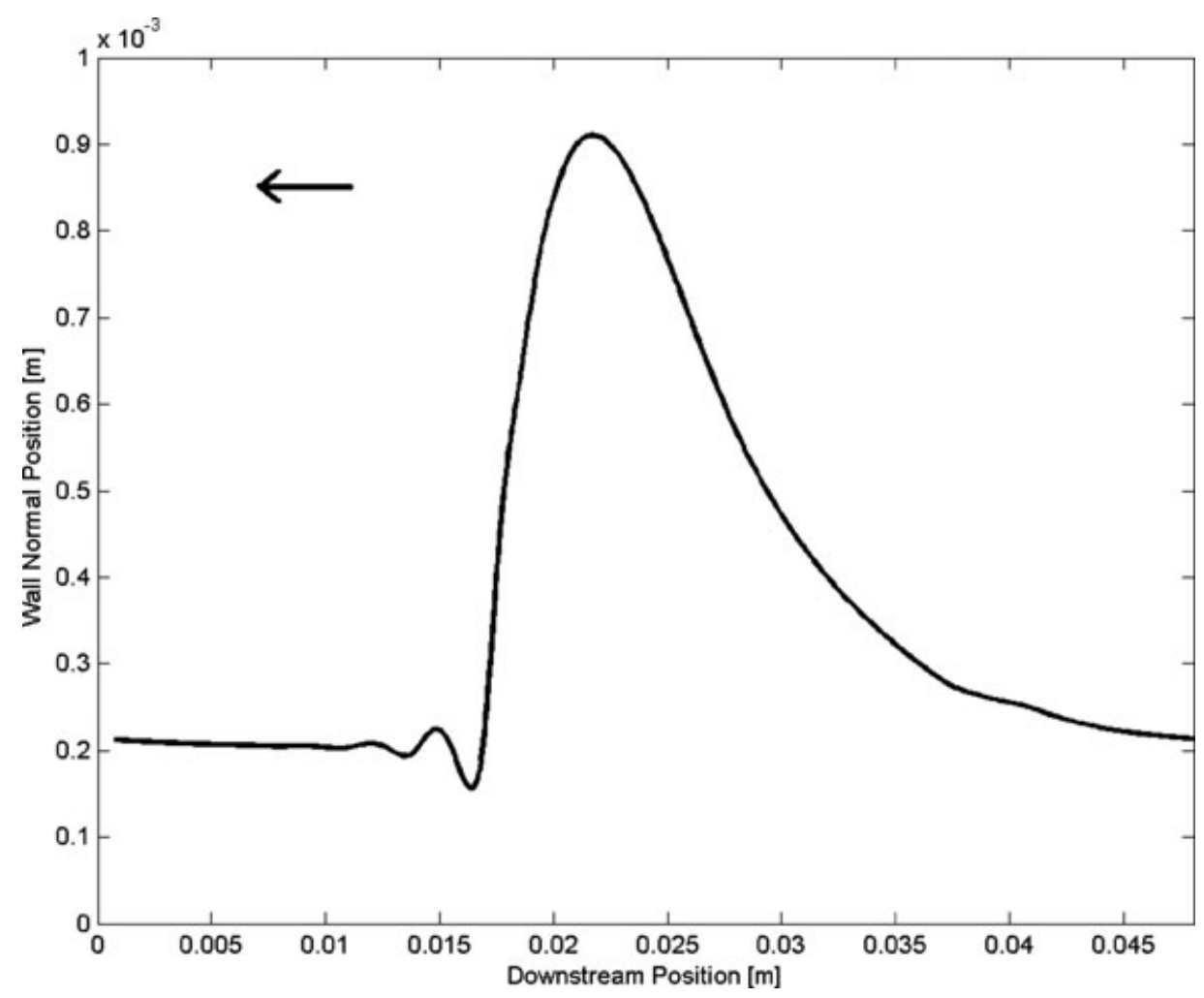

Figure 5. Typical wave profile corresponding to statistically steady state for case III.

where the initial disturbance $\epsilon \simeq h_{0} / 100$. The length of the periodic domain, Ly may have an influence on the accuracy of the solution if only one or two waves were simulated in the domain, $\left(N_{S}=1\right.$, or $\left.N_{s}=2\right)$ because the box size acts as a filter. As a result, choosing $N_{s}=4$ for all of our two-dimensional simulations shows it eliminated this effect. The nondimensional groups for this problem are defined assuming the following scales for length $\langle L\rangle$ and velocity $\langle V\rangle$. The nomenclature is chosen here to allow for a direct comparison with the results presented by Nosoko et al. [1]. Using their notation,

$$
\begin{aligned}
& \langle L\rangle \equiv\left(\frac{v^{2}}{g}\right)^{\frac{1}{3}}, \\
& \langle V\rangle \equiv(v g)^{\frac{1}{3}},
\end{aligned}
$$

we define a fluid property nondimensional group as

$$
N_{f}=\frac{\rho^{3} v^{4} g}{\sigma^{3}},
$$




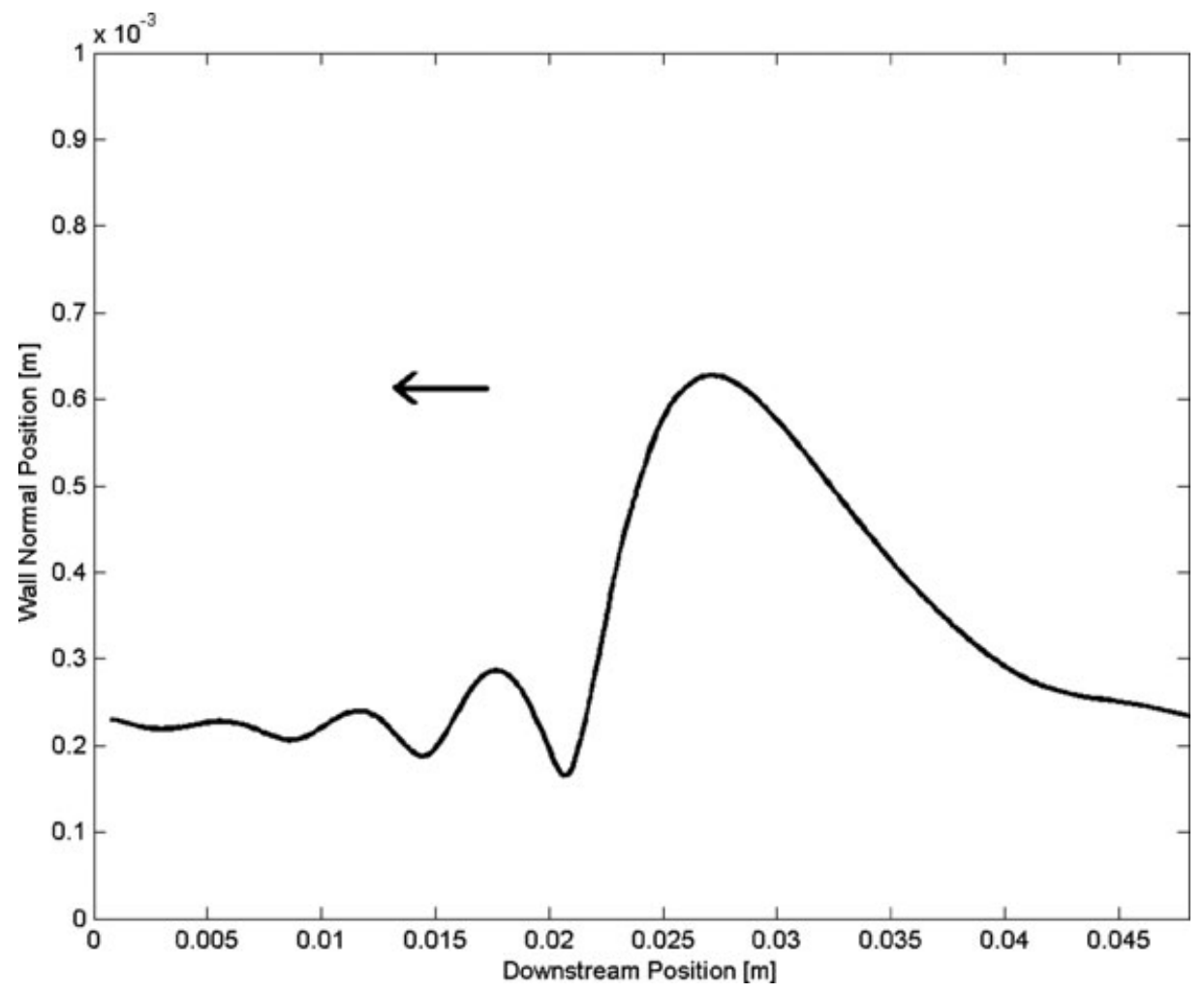

Figure 6. Typical wave profile corresponding to statistically steady state for case V.

nondimensional peak height as

$$
N_{h p}=h_{\text {peak }}\left(\frac{v^{2}}{g}\right)^{-\frac{1}{3}}
$$

nondimensional wavelength as

$$
N_{\lambda}=\lambda_{s}\left(\frac{v^{2}}{g}\right)^{-\frac{1}{3}},
$$

and, nondimensional wave velocity as

$$
N_{u w}=u_{w}(v g)^{-\frac{1}{3}} \text {. }
$$

We performed a set of six numerical experiments shown in Table 2, spanning a range of Re, $N_{f}$, and $N_{\lambda}$ similar to the experiments performed in [1].

In the regime under study in this section, the flow statistics become steady, and analysis is possible with good accuracy. For comparing with data, Nosoko et al. [1] have performed a comprehensive set of experiments in this regime, 


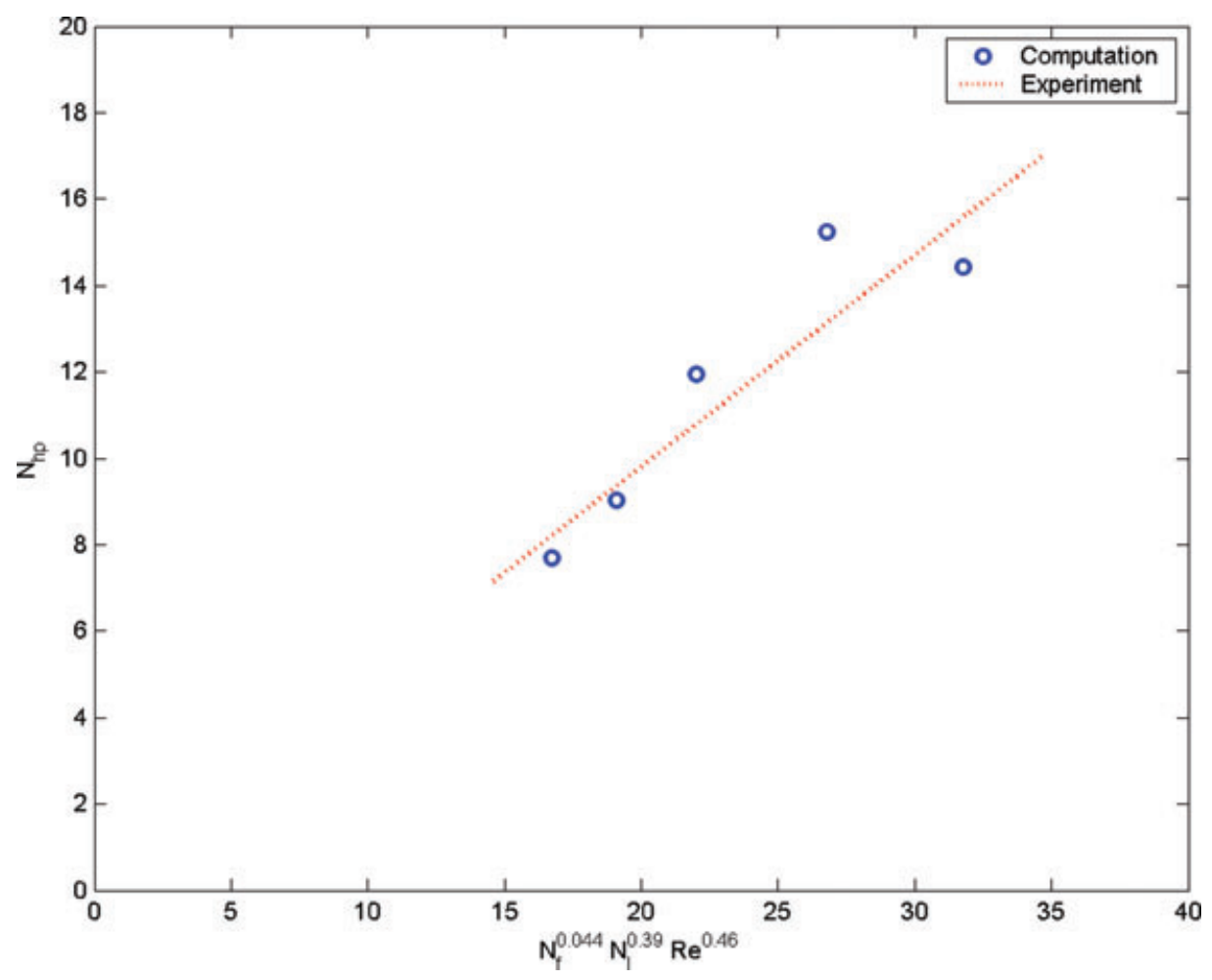

Figure 7. Comparison between nondimensional peak height experimental scaling (dashed line) and numerical experimental cases I through VI. This figure is colour online.

and were able to extract many scaling characteristics of the flow. This section will serve as a validation of our approach.

\subsection{Results}

In their work, Nosoko et al. [1] found from experiments the following relationships for wave peak height and wave velocity:

$$
\begin{aligned}
& N_{h p}=0.49 N_{f}^{0.044} N_{\lambda}^{0.39} R e^{0.46}, \\
& N_{u w}=1.13 N_{f}^{0.02} N_{\lambda}^{0.31} R e^{0.37},
\end{aligned}
$$

and

$$
N_{h p}=0.425 N_{f}^{0.019} N_{u w}^{1.25}
$$

In Figures 4-6 we present wave shapes at statistically steady state for cases II, III, and V detailed in Table 2. Although the wave profiles may match 


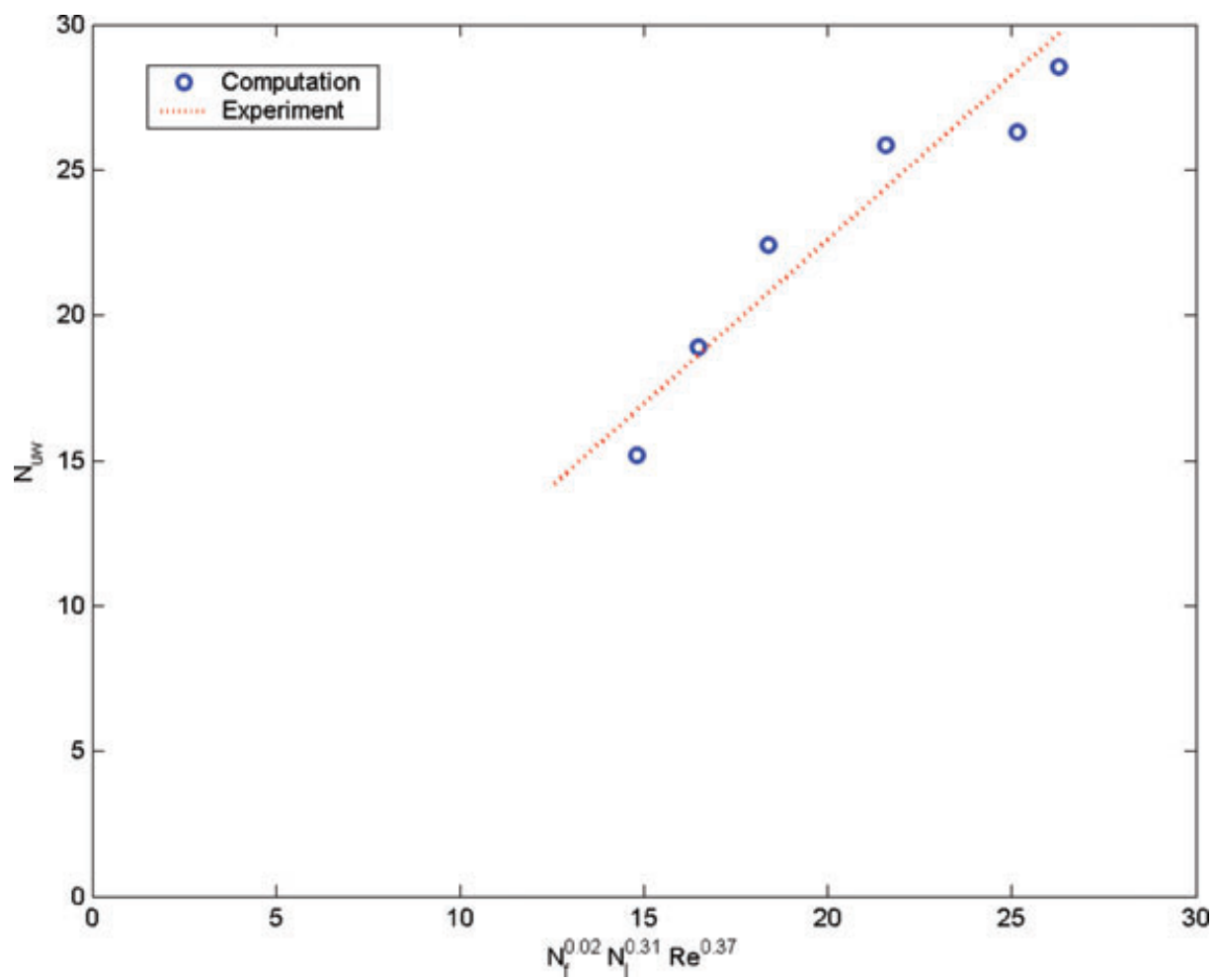

Figure 8. Comparison between nondimensional wave velocity experimental scaling (dashed line) and numerical experimental cases I through VI. This figure is colour online.

visually what is observed in experiments [8] and other numerical studies $[17,15]$, we proceed to quantify our results by comparing with empirical relationships in Equations (36)-(38).

In Figure 7, we present the scaling corresponding to (36) versus our calculations. There is good agreement of values from the simulations with the empirical relationship derived from experiment. Note at larger values of the abscissa, numerical results are more scattered. The larger scattering may be explained by the fact that for larger values of the abscissa, the film is not as statistically steady as for lower values, resulting in some difficulty in computing statistically steady film heights.

The second empirical relationship described by (37) is tested against the simulations in Figure 8. Good agreement between the relationship in [1] and the numerical simulations is again evident.

Finally, Figure 9 shows the comparison between (38) and results of the simulations. It is again evident that there is good agreement between experiments and the numerical solutions. 


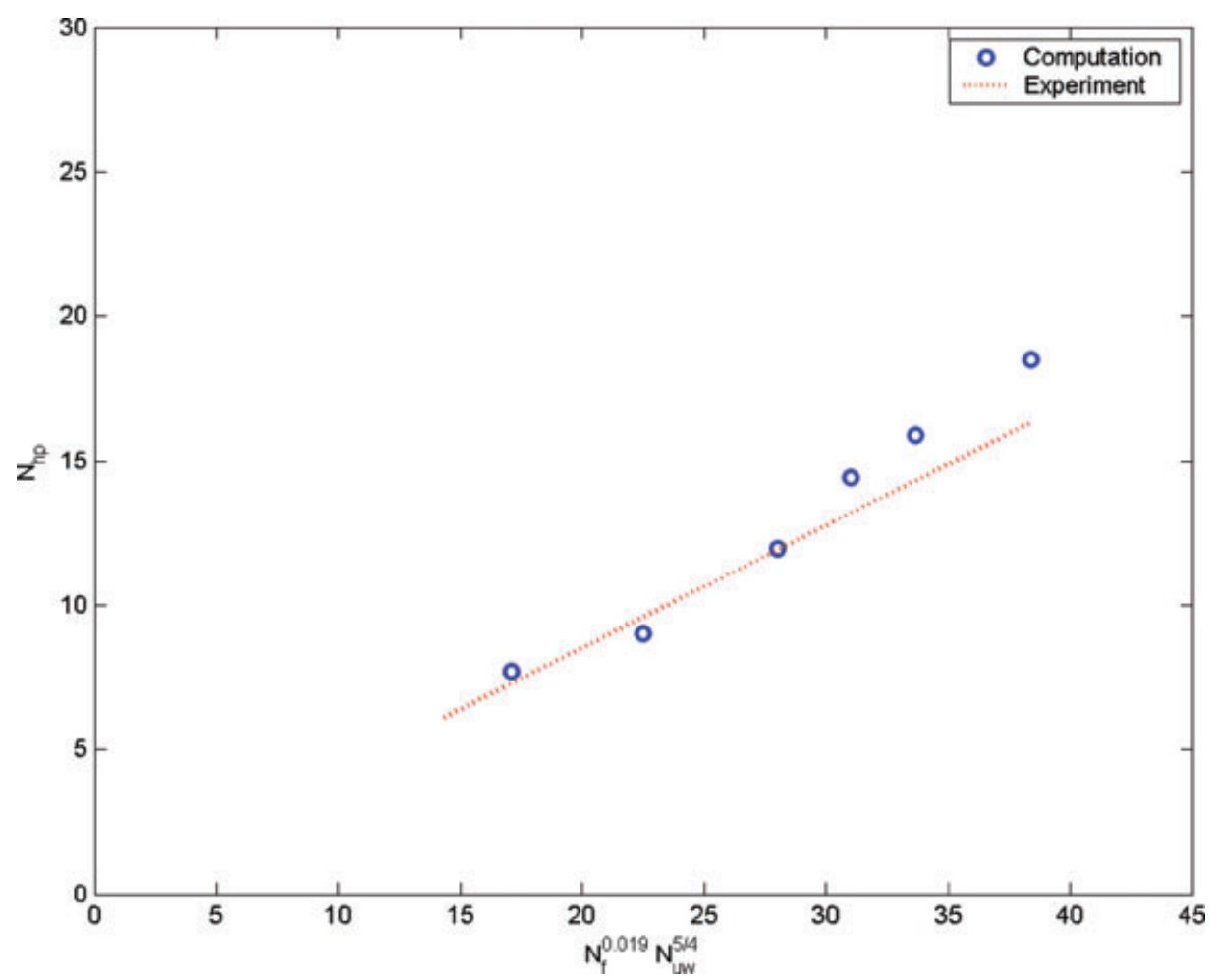

Figure 9. Comparison between nondimensional peak height experimental scaling (dashed line) and numerical experimental cases I through VI. This figure is colour online.

\section{Conclusion and outlook}

We have presented a numerical method for the Direct Numerical Simulation (DNS) of liquid films. Several issues needed to be addressed to solve the problem of liquid films with large deformation:

- The need for an elongated grid aspect ratio arising from the geometrical configuration of liquid films has warranted development of a sharp interface method based on the ghost fluid formulation [7].

- In contrast with homogeneous turbulence, liquid film turbulence is a high aspect ratio phenomenon, and again warrants using elongated grids. To perform simulations, as required for example in the DNS of high Reynolds number problems, we have developed an implicit treatment of the viscous term present in the Navier-Stokes equations thus removing the stability restriction imposed by the small grid spacing in the wall-normal direction.

- Because falling liquid films need a large domain to capture their growth to statistically steady state, we have developed a solution technique with 

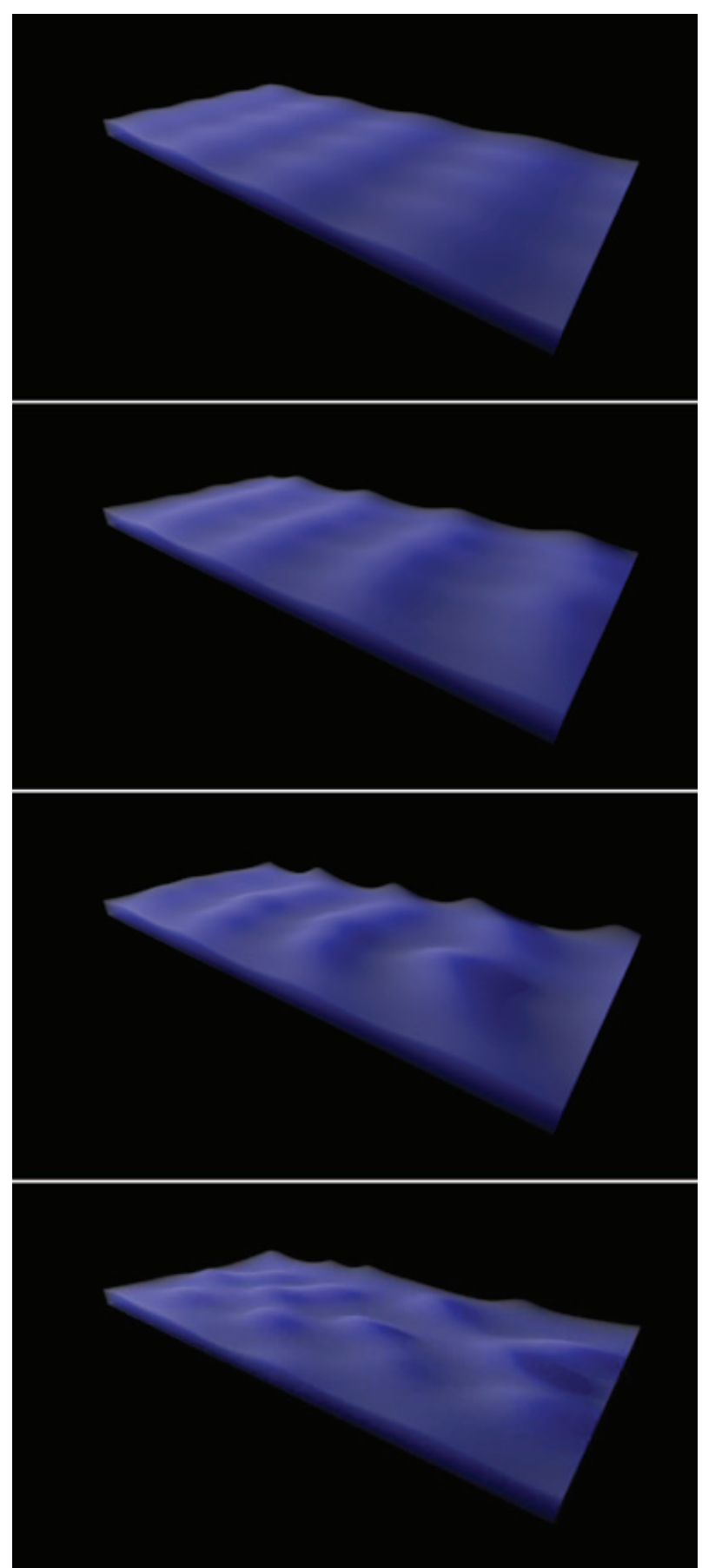

Figure 10. Transition from $2 \mathrm{D}$ to $3 \mathrm{D}$ on a vertically falling liquid film at $\mathrm{RE}=400$. (flow to the right). This figure is colour online. 


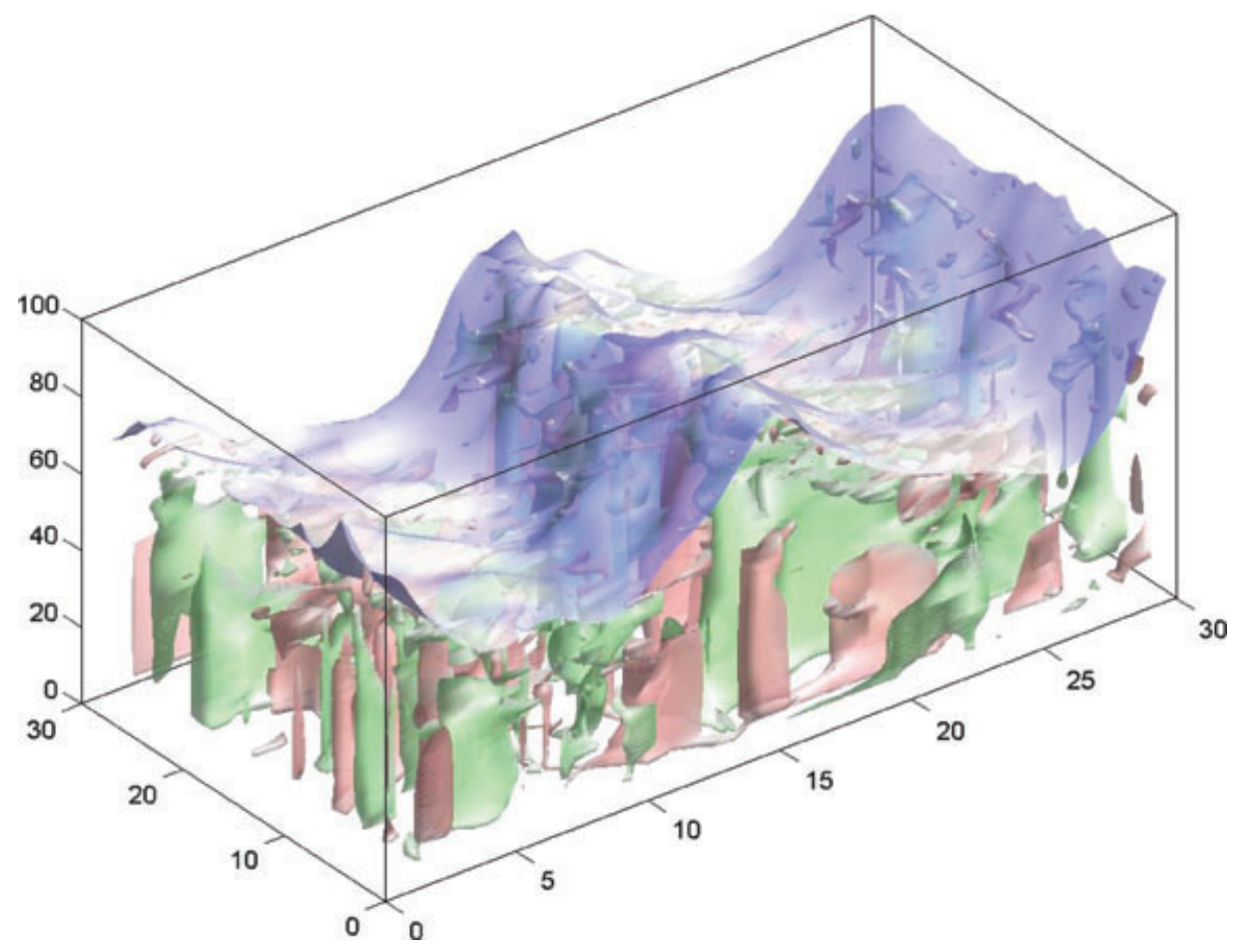

Figure 11. Structures in the liquid—No gravity/gas driven system. This figure is colour online.

periodic boundary conditions. This is also necessary for studying high Reynolds and turbulence problems, as DNS is often performed for canonical conditions using periodic boundary conditions. Figure 10 shows a sequence in time of interface profiles for a vertically falling liquid film in 3D. The flow was initialized with a 2D disturbance and we observed the transition to a fully $3 \mathrm{D}$ profile. Similar computations at much greater computational cost (the results presented in Figure 10 required a few hours on a single CPU machine) are performed in [17] using inflow/outflow conditions, thus requiring unnecessarily long domains.

- We have validated our approach by comparing our numerical simulations with the experiment of Nosoko et al. [1] for vertical falling liquid films at Reynolds number up to 100 . The empirical relationships derived from experiment were compared with our numerical calculations with good agreement. In conclusion, it is important to recognize that the numerical method developed here is very general.

As future work, we plan to study the effect of gravity on gas driven flows. As an example, Figures 11 and 12 show a case with no gravity. In Figure 11, we observe large deformation of the interface along with vortical structures in 


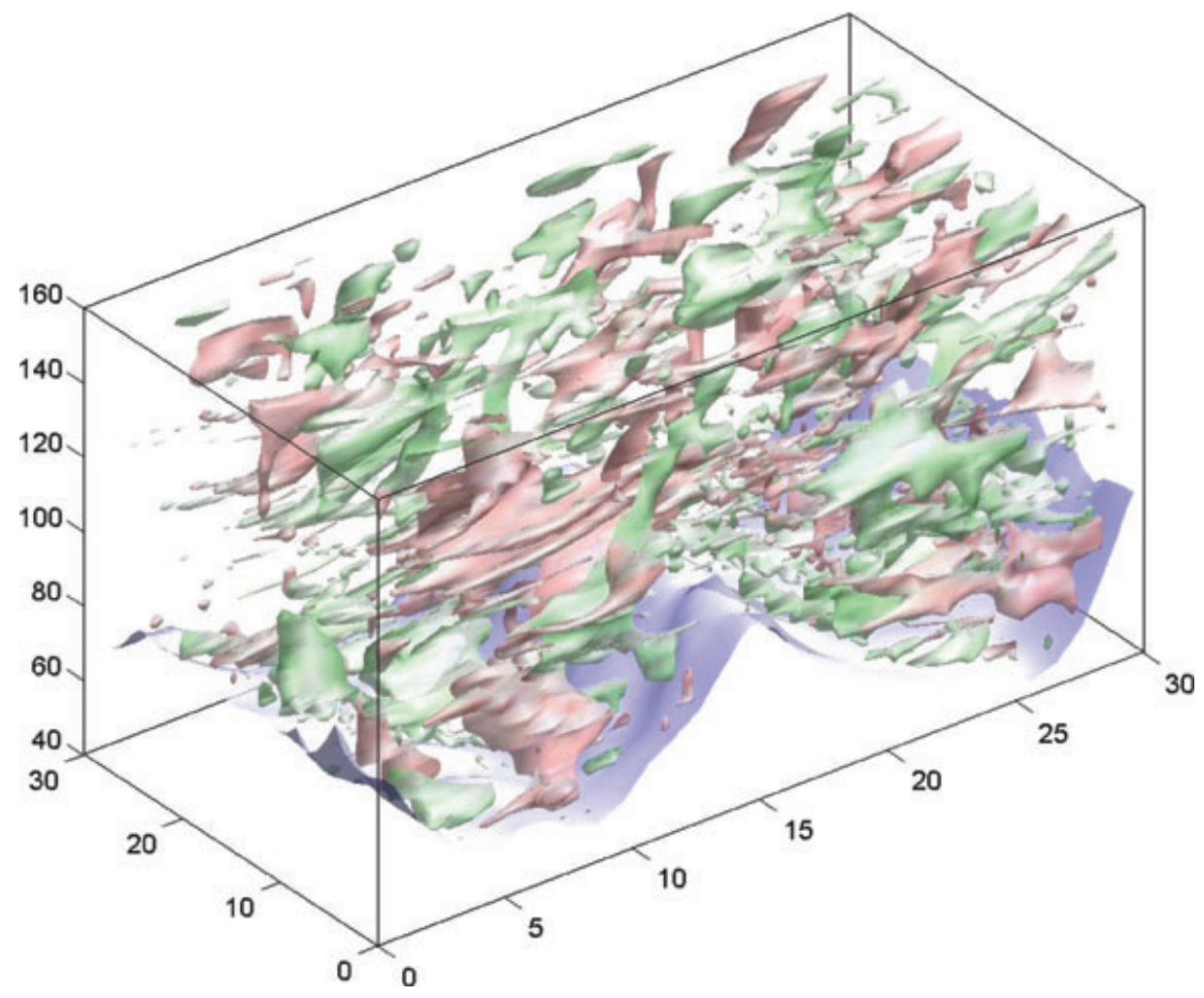

Figure 12. Vortical structures in the gas-No gravity/gas driven system. This figure is colour online.

the liquid. In Figure 12, we show vortical structures in the gas phase for the same case, and here, streamwise streaky structures are present and "wave-shape effects" on these structures is observed.

Finally, we hope the method presented here will serve as a tool to study liquid films systems whether they are gravity-driven such as falling liquid films, or gas-driven. In the latter case, a large area is now open for investigation through DNS including such systems as micro-breaking and breaking waves.

\section{Acknowledgments}

This work was partially supported by the National Aeronautics and Space Administration, Microgravity Research Division under Contract No. NAG3-2414. 


\section{References}

1. T. Nosoko, P. N. Yoshimura, et al., Characteristics of two-dimensional waves on a falling liquid film, Chem. Eng. Sci. 51(5):725-732 (1996).

2. M. Kang, R. Fedkiw, and X.-D. Liu, A Boundary Condition Capturing Method for Multiphase Incompressible Flow, UCLA CAM Report 035 (1999).

3. H. TAKAHIRA and S. BANERJEE, Numerical Simulation of three dimensional bubble growth and detachment in a microgravity shear flow, Microgravity Fluid Physics and Heat Transfer, Oahu, Hawaii, USA, 1999.

4. Y. ZANG and R. L. StReEt, A Composite Multigrid Method for Calculating Unsteady Incompressible Flows in Geometrically Complex Domains, Int. J. Numer. Meth. Fluids 20:341-361 (1995).

5. Y. ZANG and R. L. Street, A Non-staggered Grid, Fractional Step Method for Time-Dependent Incompressible Navier-Stokes Equations in Curvilinear Coordinates, J. Comp. Phys. 114:1833 (1994).

6. W. Yue, C. L. Lin, and V. C. PATEL, Numerical investigation of turbulent free surface flows using level set method and large eddy simulation, IIHR Technical Report No. 435 (2003).

7. R. Fedkiw and X.-D. LiU, The Ghost Fluid Method for Viscous Flows, Progress in Numerical Solutions of Partial Differential Equations (M. HAFEZ, Ed.), Arachon, France, 1998.

8. X.-D. Liu, R. P. Fedkiw, and M. Kang, A Boundary Condition Capturing Method for Poisson's Equation on Irregular Domains, Journal of Computational Physics 160:151-17 (2000).

9. F. H. Harlow and J. E. Welch, Numerical calculation of time-dependent viscous incompressible flow of fluid with a free surface, The Physics of Fluids 8:2182-2189 (1965).

10. A. J. Chorin, Numerical Solution of the Navier-Stokes Equations, Math. Comp. 22:745 (1968).

11. C. Canuto, M. Y. Hussaini, A. Quarteroni, and T. A. Zang, Spectral Methods in Fluid Dynamics, Springer-Verlag, New York, 1987.

12. K. Lam and S. BanerJee, On the Conditions of Streak Formation in Bounded Flows, Phys. Fluids A4:306-320 (1992).

13. K. LAM, Numerical Investigation of Turbulent Flow, Santa Barbara Ph.D. thesis, University of California, 1989.

14. C. W. HiRT and B. D. Nichols, Volume of fluid (VOF) methods for the dynamics of free boundaries, J. Comp. Phys. 39:201-25 (1981).

15. D. GaO, N. B. Morley, and V. DhiR, Numerical simulation of wavy falling film flow using VOF method, Journal of Computational Physics 192(2):624-642 (2003).

16. J. U. BRACKbill, D. B. Kothe, and C. Zemach, A continuum model for modeling surface tension, J. Comp. Phys. 100:335-354 (1991).

17. T. KUnUGi and C. KINO, DNS of Falling Film Structure and Heat Transfer via MARS Method, Comput. Struct. 83:455-462 (2005).

18. V. E. Badalassi, H. D. Ceniceros, and S. BanerJeE, Computation of multiphase systems with phase field models, J. Computat. Phys. 190:371-397 (2003).

19. X. D. Liu, S. Osher, and T. Chan, Weighted Essentially Non-Oscillatory Schemes, J. Computat. Phys. 115:200-212 (1994).

20. M. Sussman and E. Fatemi, An efficient, interface preserving level set re-distancing algorithm and its application to interfacial incompressible fluid flow, SIAM J. Sci. Comput. 20(4):1165-1191 (1999).

21. H. W. Press, S. A. Teukolsky, W. T. Vetterling, and B.P. Flannery, Numerical recipes in C, (2nd ed.), Cambridge University Press, Cambridge, UK, 1992. 
22. H. A. VAN DER VORST, Bi-CGSTAB: a fast and smoothly converging variant of Bi-CG for solution of non-symmetric linear systems, SIAM J. Sci. Stat. Comp. 13:631-644 (1992).

23. C. W. SHU and S. Osher, Efficient Implementation of Essentially Non-Oscillatory Shock Capturing Schemes, J. Computat. Phys. 88:439-471 (1988).

MassachusetTs Institute of TeChNOLOGY

University of CALIFornia, SANTA Barbara

City University of New YoRK

(Received December 10, 2009) 